\title{
The Meaning of Betti's Reciprocal Theorem
}

\section{Truesdell}

(February 5, 1963)

\begin{abstract}
It is demonstrated that Betti's reciprocal theorem represents a criterion for the existence of a stored-energy function.
\end{abstract}

It was remarked long ago $[1]^{1}$ that Betti's reciprocal theorem, familiar in the linearized theory of elasticity, remains valid for infinitesimal strain superimposed upon an arbitrarily strained state of a hyperelastic material, and recently a proof was published [2]. The true significance of Betti's theorem, however, lies in its being a criterion for the existence of a stored-energy function.

Indeed, the differential equations and stress boundary conditions to be satisfied by the superimposed displacement field $\mathbf{u}$ are [3]

$$
\left(A_{k m}{ }^{\alpha \beta} u_{; \beta}^{m}\right)_{; \alpha}=\rho_{\mathbf{R}}\left(\ddot{u}_{k}-b_{k}^{*}\right), A_{k m}{ }^{\alpha \beta} u_{; \beta}^{m} n_{\mathrm{R} \alpha}=t^{*}{ }_{\mathrm{R} k},
$$

where $\rho_{\mathrm{R}}$ is the density in the reference configuration, $\mathbf{b}^{*}$ and $\mathbf{t}_{\mathrm{R}}^{*}$ are the excesses of external body force and surface traction, respectively, above those acting on the given strained state, $\mathbf{n}_{\mathrm{R}}$ is the outward unit normal in the reference configuration, and the semicolon denotes the total covariant derivative. The coefficients $A_{k m}{ }^{\alpha \beta}$ are defined directly from the stress-strain relation of the elastic material, not assumed to be hyperelastic:

$$
T_{\mathrm{R} k^{\alpha}}=\mathfrak{h}_{k}{ }^{\alpha}\left(x^{m}, \beta\right), A_{k m}{ }^{\alpha \beta}\left(x^{p} \cdot \gamma\right)=\frac{\partial \mathfrak{h}_{k}{ }^{\alpha}}{\partial x^{m}, \beta}
$$

$\mathbf{T}_{\mathrm{R}}$ being the first Piola-Kirchhoff stress tensor [4]. In taking the stress-strain relation in the form $(2)_{1}$, we refrain from imposing the principle of material indifference, so that the results to be obtained remain valid also for theories somewhat more general than elasticity. Since an underlying deformation is laid down and fixed, the quantities $x^{p}{ }_{\gamma}$ are given functions of position. Thus the components $A_{k m}{ }^{\alpha \beta}$ are given, fixed functions of positions, and (1) is a linear differential equation for $\mathbf{u}$.

Let $\overline{\mathbf{b}}^{*}$ and $\overline{\mathbf{t}}_{\mathrm{R}}^{*}$ correspond to another infinitesimal deformation $\overline{\mathbf{u}}$ superimposed upon the same given, underlying strained state. Then

$$
\begin{gathered}
\left(A_{k m}{ }^{\alpha} \bar{u}^{m}{ }_{; \beta}\right)_{;}=\rho_{\mathrm{R}}\left(\ddot{\bar{u}}_{k}-b_{k}^{*}\right), \\
A_{k m}{ }^{\alpha \beta} \bar{u}_{;}{ }_{\beta} n_{\mathbf{R} \alpha}=\bar{t}_{\mathbf{R} k}^{*} .
\end{gathered}
$$

\footnotetext{
1 Figures in brackets indicate the literature references at the end of this paper.
}

Hence, by $(1)_{1}$,

$$
\begin{aligned}
& \rho_{\mathrm{R}}\left(\ddot{u}_{k}-b_{k}^{*}\right) \bar{u}^{k}=\left(A_{k m}{ }^{\alpha \beta} u_{: \beta}^{m}\right)_{;} \alpha \bar{u}^{k}, \\
&=\left(A_{k m}{ }^{\alpha \beta} u^{m} ; \beta\right. \\
&\left.\bar{u}^{k}\right)_{;}-A_{k m}{ }^{\alpha \beta} u_{; \beta}^{m} \bar{u}_{; \alpha}^{k} .
\end{aligned}
$$

Integration over a region $\mathscr{B}_{\mathrm{R}}$ in the reference configuration, followed by use of the divergence theorem and $(1)_{2}$, yields the following Betti identity:

$$
\begin{aligned}
\int_{\mathscr{B}_{\mathrm{R}}} \rho_{\mathrm{R}}\left(b_{k}^{*}-\ddot{u}_{k}\right) \bar{u}^{k} d V_{\mathbf{R}}+\oint_{\partial \mathscr{B}_{\mathrm{R}}} t_{\mathrm{R} k}^{*} u^{k} d S_{\mathrm{R}} \\
=\int_{\mathscr{B}_{\mathbf{R}}} A_{k m}{ }^{\alpha \beta} u_{: \beta}^{m} \bar{u}_{; \alpha}^{k} d V_{\mathbf{R}} .
\end{aligned}
$$

Betti's reciprocal theorem consists in the assertion that in the sum on the left-hand side, barred and unbarred quantities may be interchanged. In order that this be possible, for all $\mathbf{u}$ and $\overline{\mathbf{u}}$ and for all regions $\mathscr{B}_{\mathrm{R}}$, it is necessary and sufficient that

$$
A_{k m}^{\alpha \beta}=A_{m k}^{\beta \alpha},
$$

for the ground state considered.

Thus far we have laid down a single ground state. If we now demand that Betti's theorem shall hold for infinitesimal deformation from an arbitrary underlying state of strain, we infer that (6) must hold as an identity in the argument $x^{p}: \gamma$. This condition, by (2), is necessary and sufficient for the existence of a potential $\sum\left(x^{m}, \beta\right)$ such that

$$
T_{\mathrm{R} k}^{\alpha}=\frac{\partial \sum\left(x^{m}, \beta\right)}{\partial x_{, \alpha}^{k}} .
$$

Thus we have proved that Betti's reciprocal theorem, for infinitesimal deformation from an arbitrary state of strain, provides a necessary and sufficient condition that an elastic material be hyperelastic.

This result is only an interpretation of the familiar fact that a reciprocity theorem for solutions of a linear differential system is a necessary and sufficient condition for the system to be self-adjoint. 


\section{References}

[1] A. Signorini, Trasformazioni termoelastiche finite . . . , Atti XXIV Riun. Soc. Ital. Progr. Sci. 3, 6-25 (1936).

A. Signorini, Trasformazioni termoelastiche finite, Memoria 2a Annali di Mat. Pur. Appl. [4] 30, 1-72 (1949).

[2] H. Zorski, On the equations describing small deformations superposed on finite deformations, Proc. Internat. Sympos. Second-order Effects Haifa (1962).
[3] R. A. Toupin and B. Bernstein, Sound waves in deformed perfectly elastic materials. Acousto-elastic effect, J. Acoust. Soc. Am. 33, 216-225 (1961).

[4] C. Truesdell and R. A. Toupin, The Classical Field Theories, Flügge's Encyclopedia of Physies 3/1 (1960). see sec. 303 .

(Paper 67B2-95) 